\title{
AVALIAÇÃO DO CONHECIMENTO SOBRE A INFECÇÃO HIV DE ESTUDANTES DE ODONTOLOGIA ANTES E APÓS PALESTRA INFORMATIVA
}

\author{
EVALUATION OF THE DENTAL STUDENTS' KNOWLEDGE ON HIV \\ INFECTION. ANALYSIS BEFORE AND AFTER AN INFORMATIVE LECTURE
}

\author{
Maria Regina SPOSTO \\ Professora Adjunta da Faculdade de Odontologia de Araraquara UNESP. \\ Sylvia Gouveia dos SANTOS \\ Bolsista CNPq (proc. 523700/95-4 A. I.). \\ Carina DOMANESCHI \\ Estagiária da Disciplina de Diagnóstico Bucal. \\ Cláudia Maria NAVARRO \\ Professora Doutora da Disciplina de Diagnóstico Bucal da Faculdade de Odontologia UNESP. \\ Mirian Aparecida ONOFRE \\ Professora Adjunta da Faculdade de Odontologia de Araraquara UNESP.
}

$O$ conhecimento sobre a infecção HIV é essencial aos profissionais de odontologia para que estejam preparados quanto ao uso das medidas de controle da infecção cruzada. A proposta deste estudo foi avaliar os conhecimentos gerais e específicos dos alunos de graduação da FOAr-UNESP quanto à infecção HIV, antes e após assistirem a uma palestra informativa. Foram entregues na $1^{\mathrm{a}}$ etapa 160 questionários e recolhidos posteriormente. Na $2^{\mathrm{a}}$ etapa, três meses depois, foi ministrada a palestra sobre a infecção HIV com duração de 15 min e entregues 160 questionários para os mesmos alunos e recolhidos posteriormente. O índice total de acerto nos questionários preenchidos antes da palestra foi de $49 \%$ e de erro de $45,86 \%$. Após a palestra o índice de acerto foi de $54,4 \%$ e de erro $40,76 \%$. Concluímos que, mesmo após a palestra informativa, os estudantes apresentaram um índice de erro alto e pouca assimilação sobre o assunto, indicando que, apenas 15 minutos de palestra não foram suficientes para uma modificação significante no conhecimento dos estudantes. Assim, os alunos de graduação necessitam de um reforço nos conhecimentos sobre a infecção HIV, envolvendo atualização contínua dos aspectos informativos.

UNITERMOS: Síndrome de imunodeficiência adquirida; Estudantes de odontologia; Conhecimentos, atitudes e prática.

\section{INTRODUÇÃO}

O conhecimento sobre a infecção HIV é essencial aos profissionais de odontologia para um procedimento clínico seguro e de acordo com as normas de biossegurança. Estes devem estar preparados e esclarecidos quanto ao uso das medidas universais de controle de infecção cruzada nos vários ambientes de clínica $^{8}$, para prestarem atendimento sem medos irracionais $^{6,19}$.

As manifestações bucais freqüentemente são indicativos de infecção por HIV, permitindo também uma abordagem diagnóstica importante na detecção precoce da infecção por HIV. Para cumprir com estas responsabilidades e serem capazes de executar um tratamento odontológico adequado, tanto os estudantes quanto os profissionais precisam manter-se sempre atualizados ${ }^{11,21}$. 
Ainda nos dias atuais, artigos da literatura ${ }^{2,7,10}$ analisando a rotina na clínica odontológica continuam indicando o medo de cirurgiões-dentistas e estudantes de Odontologia em tratarem pacientes HIV+, sendo o motivo mais freqüente na recusa de pacientes ${ }^{5,18,20,22}$. Para que ocorram mudanças neste comportamento os aspectos informativos e educacionais, abrangendo os conhecimentos sobre a infecção pelo HIV, devem ser prioritários na formação dos estudantes de Odontologia, para que se tornem profissionais qualificados ${ }^{11,20}$.

A proposta deste trabalho foi realizar um estudo dos conhecimentos dos estudantes de graduação em Odontologia, da Faculdade de Odontologia de Araraquara - UNESP, quanto à infecção por HIV, antes e após uma palestra informativa.

\section{MATERIAIS E MÉTODOS}

Após análise e aprovação pela Comissão de Bioética os alunos do curso de graduação em Odontologia da Faculdade de Odontologia de Araraquara, foram avaliados por meio da aplicação de um questionário, composto de 40 questões, sendo 2 questões para identificação da origem do aluno (ano da graduação e sexo), 17 sobre conhecimentos gerais, 14 sobre conhecimentos específicos e 9 de ordem pessoal. O questionário foi desenvolvido pela Equipe de Pesquisa Ryan White da Universidade de Chicago-EUA ${ }^{1}$, fazendo parte de um programa de orientação, capacitação e atualização dos docentes, funcionários e estudantes quanto à infecção pelo HIV.

Esta avaliação foi realizada em dois momentos, na $1^{\mathrm{a}}$ etapa foram entregues 40 questionários em cada série do curso de graduação, num total de 160 . Foi solicitado aos estudantes a devolução do questionário sem identificação, com as questões preenchidas em um prazo máximo de

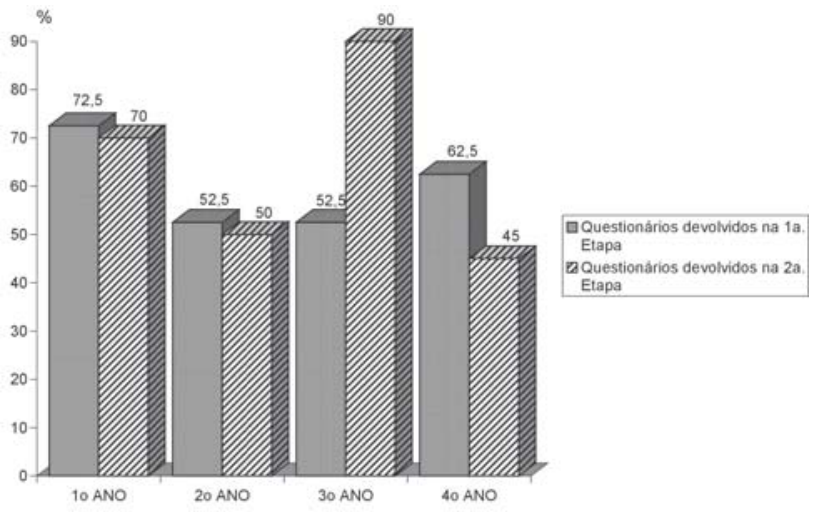

FIGURA 1- Análise da quantidade de questionários devolvidos pelos estudantes de graduação em odontologia antes ( $1^{\mathrm{a}}$ etapa) e após ( $2^{\mathrm{a}}$ etapa) palestra informativa três semanas.

Na $2^{\text {a }}$ etapa, após três meses, foi ministrada uma palestra informativa sobre a infecção HIV com duração aproximada de 15 minutos aos mesmos alunos. Esta foi realizada pela autora principal (MRS) com slides educativos sobre a infecção HIV (abordando aspectos gerais, específicos para Odontologia e de prevenção à infecção pelo HIV) e direcionadas ao esclarecimento das perguntas contidas no questionário. Logo após foram entregues 40 questionários (iguais aos da $1^{\text {a }}$ Etapa) em cada série do curso de graduação, num total de 160 e solicitado aos estudantes a devolução dos mesmos sem identificação, com as questões preenchidas em um prazo máximo de 3 semanas, como realizado na $1^{\text {a }}$ etapa.

Após a devolução, as respostas obtidas na $1^{\mathrm{a}} \mathrm{e}$ na $2^{\mathrm{a}}$ etapa foram tabuladas de acordo com um gabarito previamente elaborado pela Equipe de Pesquisa Ryan White da Universidade de Chicago - EUA ${ }^{1}$. Os resultados obtidos foram analisados através do programa Epi Info (Dean, J.; Dean, A.; Burton, A.; Dicker, R. Epi Info computer programs for epidemiology Atlanta: Center for Diseases Control and Prevention, 1990) na forma de porcentagem e números absolutos. Após tabulados, os resultados das duas etapas da pesquisa foram comparados e analisados.

\section{RESULTADOS}

Foram entregues 160 questionários, 40 em cada série, nas duas etapas da pesquisa, sendo devolvidos 96 questionários na $1^{\text {a }}$ etapa e 102 na $2^{\text {a }}$, conforme observamos na Figura 1.

Os questionários devolvidos e preenchidos foram originários, em sua maior parte, dos alunos do $1^{\circ}$ ano $(30,2 \%)$ na $1^{\mathrm{a}}$ etapa e, na $2^{\mathrm{a}}$ etapa dos alunos do $3^{\circ}$ ano $(35,30 \%)$, de acordo com a Figura 2.

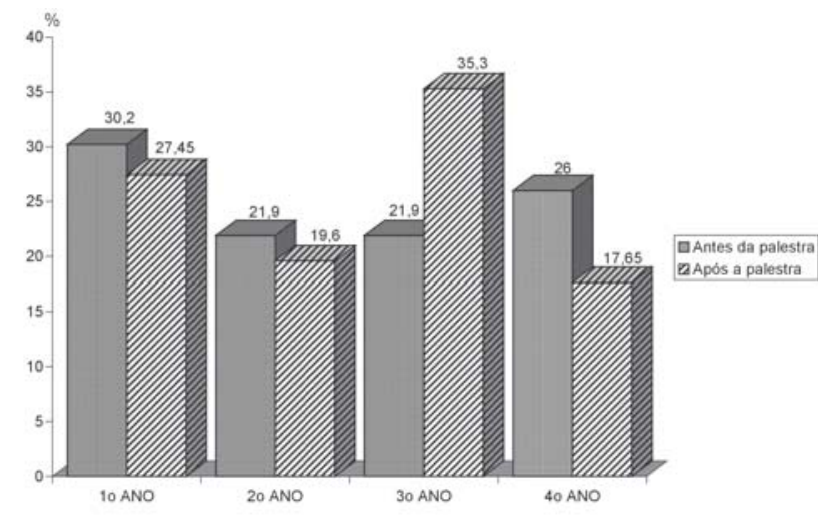

FIGURA 2- Representabilidade de cada série quanto às respostas ao questionário antes e após a palestra informativa 
As Tabelas 1, 2 e 3 analisam o índice de acertos e erros das respostas antes e após a palestra sobre a infecção HIV. Obtivemos um total de 2.976 respostas na $1^{\mathrm{a}}$ etapa, sendo que 1.632 eram de conhecimentos gerais e 1.344 de conhecimentos específicos. $\mathrm{Na} 2^{\mathrm{a}}$ etapa 3.162 respostas foram analisadas, sendo 1.734 de conhecimentos gerais e 1.428 de conhecimentos específicos.

A Tabela 2 mostra na questão 4, que somente um aluno acertou essa questão na $2^{\mathrm{a}}$ etapa, e também, que os alunos ignoram os termos: contagioso e transmissível, pois uma alta porcentagem dos alunos ( $36,5 \%$ na $1^{\text {a }}$ etapa e 34,3 na $2^{\text {a }}$ etapa), responderam erroneamente que o HIV é altamente contagioso. As questões 17 e 18, mostraram as grandes dificuldades dos alunos devido ao alto índice de erro nestas questões, além de, após a palestra, esse índice aumentar.

A Tabela 3 mostra, nas questões 25 e 29, que os alunos estão conscientes que mesmo o paciente estando numa condição imunodeprimida, ele deve manter boas condições dentárias e receber tratamentos dentários de rotina.

Analisando o índice de acertos e erros, a Tabela 1 e a Figura 3 mostram que houve um índice de acerto total do questionário, na $1^{\text {a }}$ etapa, de $49 \%$ e de erro de $45,86 \%$, com $5,14 \%$ das questões não respondidas. $\mathrm{Na} 2^{\mathrm{a}}$ etapa houve um índice de acerto total de $54,4 \%$, de erro de $40,76 \%$ sendo que $4,84 \%$ das questões não foram respondidas.

Quanto às respostas para as questões de ordem pessoal podemos observar na Tabela 4 os resultados obtidos e notamos que a metade dos alunos entrevistados, nas 2 etapas, já suspeitaram de que alguma pessoa tivesse HIV ou AIDS. A maioria dos alunos respondeu que não temem um contato social com pessoas $\mathrm{HIV}+$, porém essa mesma maioria teme tratar estes pacientes.

Nas duas etapas os alunos consideraram o questionário de média dificuldade, $68 \%$ na $1^{\text {a }}$ e $74,5 \%$ na $2^{\mathrm{a}}$ etapa. Quase a totalidade dos alunos gostaria de receber mais informações sobre a infecção HIV, na forma de conferências, seminários e palestras em grupo com oradores HIV+.

\section{DISCUSSÃO}

Levando-se em conta que, para a realização deste estudo, foi ministrada uma palestra informativa sobre a infecção HIV, a qual continha todos os tópicos que respondiam direta ou indiretamente o questionário aplicado, esperamos que na $2^{\mathrm{a}}$ etapa da pesquisa fosse observada uma melhoria no índice de acertos. Isso não foi observado, pois o índice total de acerto foi de $49 \%$

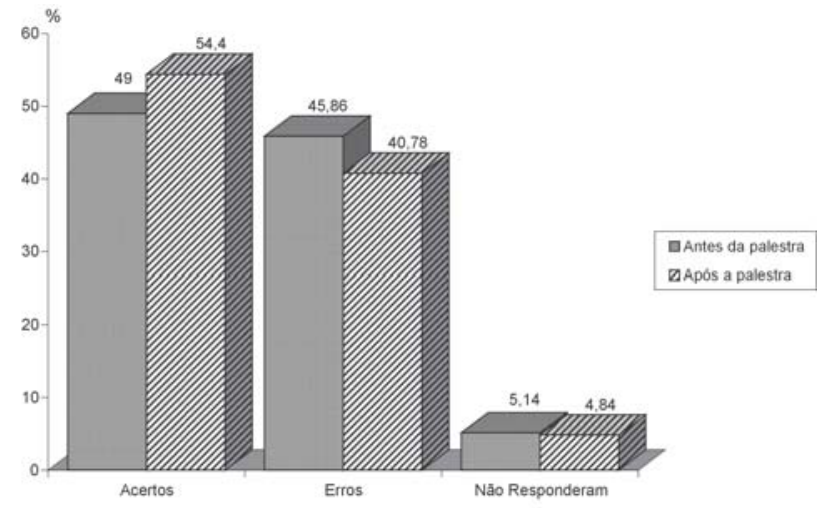

FIGURA 3- Índice total de acertos e erros nos questionários preenchidos de acordo com abordagem das questões antes e após palestra informativa

TABELA 1- Índice de acertos e erros nos questionários preenchidos de acordo com a abordagem das questões antes e após palestra informativa

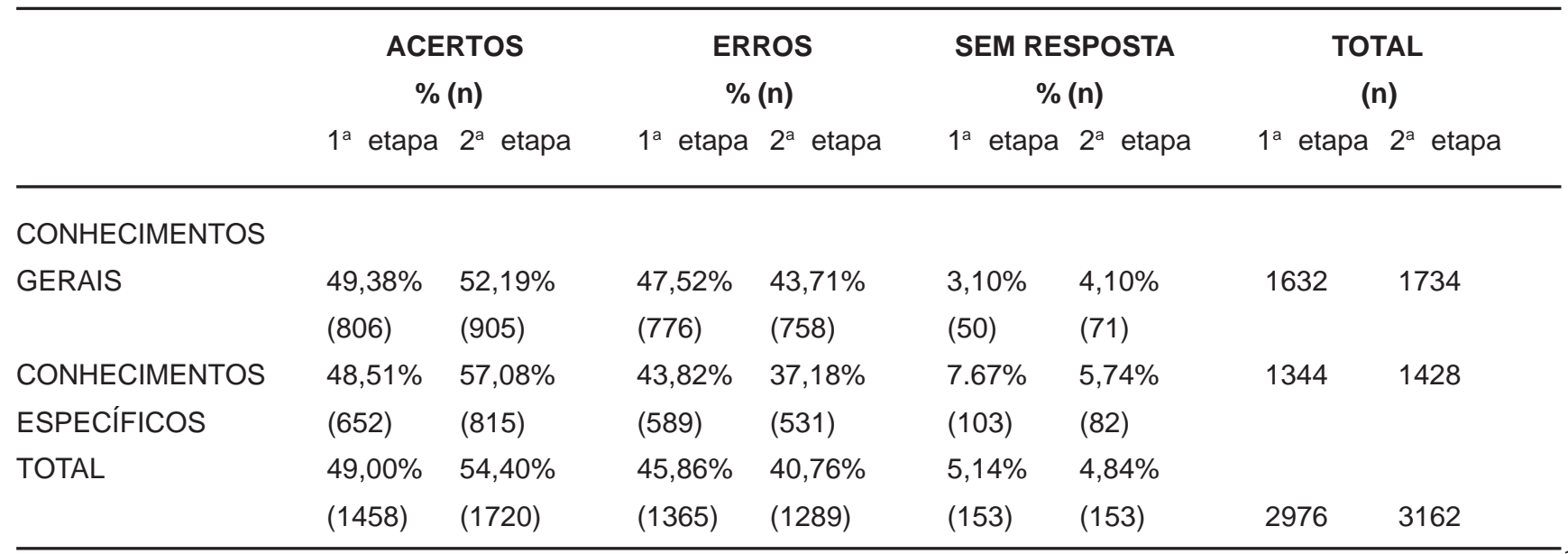


antes da palestra e 54,4\% após a palestra, ou seja, não houve um aumento marcante nas porcentagens de acerto, contrariando nossas expectativas. Além disso, em algumas questões o índice de erro foi maior após a palestra apesar da maioria dos alunos (74\%) considerarem o questionário de média dificuldade.

Os resultados encontrados na Universidade de Chicago $^{1}$, onde foi aplicado o mesmo questionário, mostraram uma maior coerência, pois o índice de acerto foi de $44 \%$ mas, a maioria dos alunos (34\%) considerou o questionário difícil e $11 \%$ o consideraram muito difícil. Em ambas universidades a maioria dos alunos demonstrou interesse em obter mais informações sobre a infecção HIV. Na $1^{\text {a }}$ etapa do trabalho houve uma maior participação dos alunos do $1^{\circ}$ ano porém, depois de ministrada a palestra o retorno maior foi do $3^{\circ}$ ano. Talvez seja porque esses alunos estejam iniciando uma intensa atividade clínica gerando entre eles uma maior preocupação quanto aos procedimentos de biossegurança.

Para maior facilidade na análise dividimos a discussão dos resultados em três seguimentos: conhecimentos gerais, específicos e questões de ordem pessoal.

\section{Conhecimentos gerais}

A $2^{\mathrm{a}}$ etapa da pesquisa mostra resultados com quase a totalidade dos alunos $(98,0 \%)$ conseguindo assimilar que a infecção por HIV pode acometer qualquer pessoa, demonstrando que a palestra acabou com a idéia preconceituosa de que o HIV é uma infecção que atinge somente um certo grupo de pessoas (homossexuais, usuários de drogas e prostitutas).

TABELA 2- Índices de acertos e erros nas questões sobre conhecimentos gerais do questionário antes e após palestra informativa

\begin{tabular}{|c|c|c|c|c|c|c|}
\hline \multirow[t]{2}{*}{ Questões } & \multicolumn{2}{|c|}{$\begin{array}{l}\text { Acertos } \\
\%(n)\end{array}$} & \multicolumn{2}{|c|}{$\begin{array}{r}\text { Erros } \\
\%(n)\end{array}$} & \multicolumn{2}{|c|}{$\begin{array}{c}\text { Sem Resposta } \\
\%(n)\end{array}$} \\
\hline & $1^{\text {a }}$ etapa & $2^{a}$ etapa & $1^{\text {a }}$ etapa & $2^{a}$ etapa & $1^{\mathrm{a}}$ etapa & $2^{a}$ etapa \\
\hline 3) Qual grupo de pessoas o HIV acomete? & $41,7(40)$ & $98,0(100)$ & $36,4(35)$ & $1,0(1)$ & $21,9(21)$ & $1,0(1)$ \\
\hline $\begin{array}{l}\text { 4) Numere de } 1 \text { a } 9 \text { os meios mais efetivos } \\
\text { para transmissão do HIV. }\end{array}$ & 0 & $1,0(1)$ & $100(96)$ & $99(101)$ & 0 & 0 \\
\hline 5) O maior índice de transmissão é? & $50,0(48)$ & $49,0(50)$ & $50,0(48)$ & $51,0(52)$ & 0 & 0 \\
\hline $\begin{array}{l}\text { 6) Qual fluído corpóreo carrega a maior e } \\
\text { menor quantidade de vírus HIV? }\end{array}$ & $36,5(35)$ & $32,4(33)$ & $62,5(60)$ & $57,2(59)$ & $1,0(1)$ & $9,8(10)$ \\
\hline $\begin{array}{l}\text { 7) A AIDS é o maior problema de saúde } \\
\text { pública? }\end{array}$ & $41,7(40)$ & $45,1(46)$ & $58,3(56)$ & $52,0(53)$ & 0 & $2,9(3)$ \\
\hline $\begin{array}{l}\text { 8) Pacientes c/ AIDS são tão infecciosos quanto } \\
\text { pacientes HIV positivos? }\end{array}$ & $86,5(83)$ & $80,4(82)$ & $13,5(13)$ & $16,7(17)$ & 0 & $2,9(3)$ \\
\hline 9) Em que período a pessoa é mais infectante? & $36,5(35)$ & $30,4(31)$ & $56,3(54)$ & $52,9(54)$ & $7,3(7)$ & $16,7(17)$ \\
\hline 10)O HIV é altamente contagioso? & $63,5(61)$ & $64,7(66)$ & $36,5(35)$ & $34,3(35)$ & 0 & $1,0(1)$ \\
\hline 11) O HIV é transmissível, mas não contagioso? & $69,8(67)$ & $64,7(66)$ & $29,2(28)$ & $34,3(35)$ & $1,0(1)$ & $2,9(3)$ \\
\hline $\begin{array}{l}\text { 12)O HIV é altamente transmissível e } \\
\text { contagioso? }\end{array}$ & $66,7(64)$ & $63,7(65)$ & $32,3(31)$ & $31,4(32)$ & $1,0(1)$ & $4,9(5)$ \\
\hline 13) Assinale a sentença correta. & $74,0(71)$ & $87,3(89)$ & $24,0(23)$ & $12,8(13)$ & $2,1(2)$ & 0 \\
\hline 14) Qual a taxa média de sobrevida de pac. HIV+ & $62,5(60)$ & $59,8(61)$ & $34,4(33)$ & $36,3(37)$ & $3,1(3)$ & $3,9(4)$ \\
\hline 15)O que o HIV pode causar? & $7,3(7)$ & $13,7(14)$ & $84,4(81)$ & $80,4(82)$ & $8,3(8)$ & $5,9(6)$ \\
\hline $\begin{array}{l}\text { 16) Os pac. HIV+ têm } 10 x \text { mais chances de } \\
\text { desenvolverem tumores malignos? }\end{array}$ & $60,4(58)$ & $69,6(71)$ & $39,6(38)$ & $29,4(30)$ & 0 & $1,0(1)$ \\
\hline 17) Quais as causas de morte entre pac. HIV+? & $31,3(30)$ & $26,5(27)$ & $67,7(65)$ & $71,5(72)$ & $1,0(1)$ & $2,0(2)$ \\
\hline $\begin{array}{l}\text { 18) Quais as causas de contaminação dos } \\
\text { recém-nascidos? }\end{array}$ & $22.9(22)$ & $18,6(19)$ & $77,1(74)$ & $77,5(79)$ & 0 & $3,9(4)$ \\
\hline 19) Quem deveriam ser os doadores de sangue? & $88,5(85)$ & $81,4(83)$ & $6,2(6)$ & $7,9(8)$ & $5,2(5)$ & $10,8(11)$ \\
\hline
\end{tabular}


Em pesquisa com estudantes do $2^{\circ}$ ano de Medicina da Universidade de Nebraska (USA) ${ }^{13}$ os resultados demonstraram que os estudantes que têm amigos homossexuais ou HIV+ são significantemente mais tolerantes com pacientes HIV+.

\section{Conhecimentos específicos}

Uma pesquisa realizada em 16 Campi das Faculdades de Odontologia no Reino Unido ${ }^{11}$ concluiu que, entre os alunos do $4^{\circ}$ ano de graduação, houve uma deficiência significante nos diagnósticos das

TABELA 3- Índices de acertos e erros nas questões sobre conhecimentos específicos do questionário antes e após palestra informativa

\begin{tabular}{|c|c|c|c|c|c|c|}
\hline \multirow[t]{2}{*}{ Questões } & \multicolumn{2}{|c|}{$\begin{array}{l}\text { Acertos } \\
\%(n)\end{array}$} & \multicolumn{2}{|c|}{$\begin{array}{r}\text { Erros } \\
\%(n)\end{array}$} & \multicolumn{2}{|c|}{$\begin{array}{l}\text { Sem Resposta } \\
\%(n)\end{array}$} \\
\hline & $1^{\text {a }}$ etapa & $2^{\mathrm{a}}$ etapa & $1^{\text {a }}$ etapa & $2^{\mathrm{a}}$ etapa & $1^{\text {a }}$ etapa & $2^{\mathrm{a}}$ etapa \\
\hline $\begin{array}{l}\text { 20) Sarcoma de Kaposi é um câncer causado por } \\
\text { um vírus? }\end{array}$ & $35,4(34)$ & $49,0(50)$ & $52,1(50)$ & $44,1(45)$ & $12,5(12)$ & $6,9(7)$ \\
\hline $\begin{array}{l}\text { 21) Leucoplasia pilosa é uma infecção que ameaça } \\
\text { a vida? }\end{array}$ & $66,7(64)$ & $82,4(84)$ & $24,0(23)$ & $15,7(16)$ & $9,4(9)$ & $2,0(2)$ \\
\hline 22)Candidíase pode matar? & $15,6(15)$ & $7,8(8)$ & $78,1(75)$ & $91,2(93)$ & $6,3(6)$ & $1,0(1)$ \\
\hline $\begin{array}{l}\text { 23)É importante que pac. HIV+ mantenham uma } \\
\text { boa higiene bucal? }\end{array}$ & $87,5(84)$ & $91,2(93)$ & $12,5(12)$ & $6,9(7)$ & 0 & $2,0(2)$ \\
\hline 25)O dentista pode transmitir HIV para os pac.? & $77,1(74)$ & $72,5(74)$ & $22,9(22)$ & $25,5(26)$ & 0 & $2,0(2)$ \\
\hline 26)O dentista pode contrair HIV de um pac.? & $96,9(93)$ & $97,1(99)$ & $3,1(3)$ & $2,9(3)$ & 0 & 0 \\
\hline 27.1)Se sim, quais as chances? & $16,7(16)$ & $59,8(61)$ & $75,1(72)$ & $35,3(36)$ & $8,3(8)$ & $4,9(5)$ \\
\hline 28)Como você deve atender um pac. HIV+? & $10,4(10)$ & $18,6(19)$ & $46,9(45)$ & $39,2(40)$ & $42,7(41)$ & $42,2(43)$ \\
\hline 29)Qual tratamento deve receber os pac. HIV+? & $95,8(92)$ & $95,1(97)$ & $2,0(2)$ & $2,9(3)$ & $2,1(2)$ & $2,0(2)$ \\
\hline $\begin{array}{l}\text { 30)Pac. HIV+ devem ser pré-medicados c/ } \\
\text { antibióticos por causa da imunodepressão? }\end{array}$ & $31,3(30)$ & $42,2(43)$ & $65,6(63)$ & $54,9(56)$ & $3,1(3)$ & $2,9(3)$ \\
\hline $\begin{array}{l}\text { 31)Pac. HIV + devem ser pré-medicados c/ } \\
\text { antibióticos por causa do estágio da doença? }\end{array}$ & $51,0(49)$ & $55,9(57)$ & $43,8(42)$ & $41,2(42)$ & $5,2(5)$ & $2,9(3)$ \\
\hline 32)Como proceder com o instrumental utilizado? & $60,4(58)$ & $78,4(80)$ & $34,4(33)$ & $18,6(19)$ & $5,2(5)$ & $2,9(3)$ \\
\hline $\begin{array}{l}\text { 33)Abcessos dentais, úlceras, cáries são mais } \\
\text { prováveis de ocorrerem em pac. HIV+? }\end{array}$ & $28,1(27)$ & $28,4(29)$ & $67,7(65)$ & $69,6(71)$ & $4,2(4)$ & $2,0(2)$ \\
\hline $\begin{array}{l}\text { 34)Correlacione as manifestações bucais mais } \\
\text { comumente relacionadas com o HIV. }\end{array}$ & $6,3(6) 20$, & $6(21)$ & $85,4(82)$ & $72,5(74)$ & $8,3(85)$ & $6,9(7)$ \\
\hline
\end{tabular}

TABELA 4 -Respostas de ordem pessoal dos alunos de graduação da FOAr, antes e após palestra informativa

\begin{tabular}{|c|c|c|c|c|c|c|}
\hline \multirow[t]{3}{*}{ Questões de ordem pessoal } & \multirow{2}{*}{\multicolumn{2}{|c|}{$\begin{array}{l}\text { SIM } \\
\%(n)\end{array}$}} & \multirow{2}{*}{\multicolumn{2}{|c|}{$\begin{array}{l}\text { NÃO } \\
\%(n)\end{array}$}} & \multirow{2}{*}{\multicolumn{2}{|c|}{$\begin{array}{c}\text { SEM RESPOSTA } \\
\%(\mathrm{n})\end{array}$}} \\
\hline & & & & & & \\
\hline & $1^{\mathrm{a}}$ etapa & $2^{\mathrm{a}}$ etapa & $1^{\mathrm{a}}$ etapa & $2^{\mathrm{a}}$ etapa & $1^{\mathrm{a}}$ etapa & $2^{\mathrm{a}}$ etapa \\
\hline Suspeitou de alguém ter HIV ou AIDS? & $51,0(49)$ & $50,0(51)$ & $47,9(46)$ & $50,0(51)$ & $1,0(1)$ & 0 \\
\hline Tem medo do contato social com pessoas HIV+? & $20,8(20)$ & $26,5(27)$ & $78,1(75)$ & $73,5(75)$ & $1,0(1)$ & 0 \\
\hline Já tratou pac. HIV+? & $12,5(12)$ & $18,6(19)$ & $85,4(82)$ & $79,4(81)$ & $2,1(2)$ & $2,0(2)$ \\
\hline Teme tratar de pac. HIV+? & $60,4(58)$ & $53,9(55)$ & $39,6(38)$ & $46,1(47)$ & 0 & 0 \\
\hline Gostaria de receber mais informações? & $96,9(93)$ & $98,0(100)$ & $2,1(2)$ & $1,0(1)$ & $1,0(1)$ & $1,0(1)$ \\
\hline
\end{tabular}


lesões bucais associadas ao HIV o que pode acarretar um inadequado diagnóstico e tratamento. Notamos essa deficiência nos alunos de graduação, devido ao alto índice de erros nas questões 22, 33 e 34 que estão relacionadas com as manifestações bucais associadas ao HIV.

A questão 28, em que foi interrogado aos alunos como proceder no atendimento de um paciente $\mathrm{HIV+}$, nos chamou a atenção, pois mesmo tendo sido exposto na palestra e em literatura ${ }^{6,8}$ que as normas de biossegurança devem ser utilizadas para todos os pacientes, sem distinção, somente $18,6 \%$ dos alunos responderam corretamente. A maioria ignora que se deve tratar os pacientes HIV+ com os mesmos cuidados que qualquer paciente. Do total de alunos $42,2 \%$ não responderam essa questão e $39,2 \%$ responderam que deveriam tomar mais precauções de segurança com esses pacientes. Isso é incoerente, pois alguns pacientes podem ser infectados pelo HIV, ou estarem com doenças infecto-contagiosas e desconhecerem ou esconderem o fato ${ }^{4}$.

Uma pesquisa realizada no Canadá ${ }^{4}$ mostra que $25 \%$ dos pacientes HIV+ entrevistados nunca relataram tal fato aos seus cirurgiões-dentistas, entretanto $83 \%$ deles gostariam que os mesmos soubessem do seu estado de saúde. Esse fato aumenta a ênfase quanto à precaução universal para todos os pacientes. No Japão ${ }^{12}$, um estudo concluiu que para o correto aprendizado sobre a infecção HIV, os profissionais e os estudantes de Odontologia, devem ter um treinamento sobre aspectos médicos e psicológicos para que possam promover um tratamento adequado aos pacientes $\mathrm{HIV}+$.

\section{Questões de ordem pessoal}

No Reino Unido há uma garantia do governo para o atendimento odontológico aos pacientes HIV+, responsabilizando todos os cirurgiões-dentistas a tratarem esses pacientes e, se o paciente tiver dificuldade em obter o tratamento deve avisar imediatamente o Serviço de Saúde Familiar ${ }^{10}$. Este procedimento também é observado no Canadá9. No Brasil, não há nenhuma lei específica que obrigue os cirurgiões-dentistas a tratarem pacientes $\mathrm{HIV+}$, porém se este se recusar pode responder a um processo por discriminação.

A literatura mostra que existe medo dos estudantes ou mesmo dos cirurgiões-dentistas em atender pacientes $\mathrm{HIV}^{2,5,7,11,18,20}$. Porém, essa mesma literatura indica que os estudantes e os profissionais não demonstram essa aversão quando se trata da hepatite $\mathrm{B}$, doença tão ou mais preocupante que a AIDS. Os meios de transmissão da hepatite B são praticamente os mesmos e esta doença têm sido transmitida com maior freqüência em consultórios odontológicos que o HIV por causa do seu alto grau de contágio 5 . Estudo realizado na cidade do México ${ }^{17}$ mostra que a conscientização dos cirurgiõesdentistas quanto ao risco de infecção por hepatite B é maior que os riscos de infecção por HIV, porém, $78 \%$ dos profissionais analisados na pesquisa não estavam imunizados contra a hepatite B.

Cirurgiões-dentistas mais jovens têm maior disposição para atender pacientes HIV $+{ }^{16}$. Por outro lado, cirurgiõesdentistas que possuem atividades em seu consultório particular têm atitudes negativas, quando comparados com cirurgiões-dentistas que atuam também no setor público, quanto ao tratamento de pacientes $\mathrm{HIV}+{ }^{7,22}$.

Em estudo realizado na Universidade Nacional Autónoma de México ${ }^{18} 82,9 \%$ dos entrevistados responderam ter consciência que é um dever ético tratar de pacientes $\mathrm{HIV+}$, porém 32,9\% disseram que não atenderiam um paciente se soubessem que este é portador do vírus. Em Singapura ${ }^{3}$, médicos também acreditam no dever ético em tratar pacientes HIV+ e este estudo, mostrou que os cirurgiões-dentistas de Singapura estão mais informados que os médicos sobre a infecção HIV e 95\% utilizam práticas universais de prevenção para qualquer paciente.

Em nossa amostra de estudantes, 53,9\% mesmo após a palestra, responderam temer tratar de pacientes HIV+, $40,7 \%$ indicaram o medo de contrair a doença, como o principal motivo. A maioria deles, $59,8 \%$, acertou que as chances do dentista contrair a doença são de 1:1.000.000 em procedimentos odontológicos desde que se tome todas as medidas de biossegurança. Uma vez que é dever de todos os cirurgiões-dentistas utilizar-se das medidas de biossegurança para todos os pacientes não deveria haver nenhum temor pelo fato do paciente ter se identificado como portador do HIV. Nesse aspecto, os resultados da pesquisa são coerentes, pois os estudantes temem tratar pacientes HIV+ porque não têm um conhecimento sólido sobre a infecção HIV ${ }^{15}$, portanto, não adquiriram auto-confiança para o tratamento desses pacientes.

Na Universidade da Pensilvânia ${ }^{14}$ um estudo mostrou ser grande a motivação de estudantes voluntários para o tratamento de pacientes com doenças infecciosas. Todos os entrevistados demonstraram interesse pela área de Medicina Oral sendo todos alunos do $4^{\circ}$ ano que fizeram um curso optativo completo sobre HIV/AIDS. Esses estudantes declararam que preferem trabalhar em clínicas de Medicina Oral com pacientes infectados do que em outras clínicas, pois esses pacientes são mais motivados e receptivos. 


\section{CONCLUSÕES}

A partir dos resultados dessa pesquisa concluímos que, mesmo após uma palestra informativa, os estudantes apresentaram um alto índice de erro e pouca assimilação sobre o assunto, demonstrando que apenas 15 minutos de palestra não são suficientes para a fixação do assunto. Mostrou também que os estudantes pensam que sabem bastante sobre a infecção HIV, já que a maioria $(74,5 \%)$ considerou o questionário de média dificuldade, mas os índices de erros foram altos. Assim, os estudantes de graduação necessitam de uma maior amplitude do conhecimento sobre a infecção HIV, envolvendo também a atualização contínua dos aspectos informativos.

\section{AGRADECIMENTOS}

Agradecemos ao apoio financeiro dado pelo $\mathrm{CNPq}$ (IC-523700/95-4AI) e a C.D. Carolina Pieroni pelo auxílio na coleta de dados realizada na $1^{\text {a }}$ etapa da pesquisa. Agradecimentos Sr. Antonio Medeiros Filho pelo trabalho de digitação do texto e adequação das Figuras e Tabelas.

\section{ABSTRACT}

The knowledge about HIV infection and AIDS is essential to the dentist. So they should be prepared in the use of measures of cross infection control. The purpose of this research is to evaluate the general and specific knowledge about HIV infection, of the dental students before and after an informative lecture on the subject. In the first stage of the evaluation 160 questionnaires, were given to the students. In the second stage, 3 months later, they went to a 15 minutes lecture about the HIV infection, afterwards another 160 questionnaires were given to the same students. In the questionnaires filled before the lecture the total success index was of $49 \%$ and of mistakes was $45.9 \%$. After the lecture those values were $54.4 \%$ and $40.8 \%$ respectively. We concluded that even after the informative lecture the students presented a high mistake index and little assimilation of the subject, indicating that just 15 minutes of lecture was insufficient for a subject of great importance. The graduation dental students need more information on the HIV infection as well as up to date informative aspects.

UNITERMS: Adquired immunodeficiency syndrome; Dental students, Knowledge, attitudes and practice.

\section{REFERÊNCIAS}

1- Alves MC, Allen T, Alves MEAF. Evaluation of residual knowledge on HIV disease. J Dent Res 1997; 76: 211.

2- Angelillo IF, Villari P, Derrico, MM, Grasso GM, Ricciardi G, Pavia M. Dentists and AIDS: a survey of knowledge, attitudes, and behavior in Italy. J Public Health Dent 1994; 54: 145-52.

3- Chan R, Khoo L, Goh CL, Lam MS. A knowledge, attitudes, beliefs and practices (KABP) survey on HIV infection and AIDS among doctors and dental surgeons in Singapore. Ann Acad Med Singapore, 1997; 26: 581-7.

4- Charbonneau A, Maheux B, Beland F. Do people with HIV/ AIDS disclose their HIV-positivity to dentists? AIDS Care 1999; 11: 61-70.

5- Chehaitly A, Alary M. Knowledge, attitudes, and professional behaviors of third- and fourth- year dental students concerning AIDS and Hepatitis B. J Dent Educ 1995; 59: 844-9.

6- Cunha VJ, Rocha SC, Onofre MA, Campos AA, Sposto MR. Avaliação do controle da infecção cruzada nas Clínicas de Graduação do Curso de Odontologia. Rev Odontol da UNESP 1997; 26: 307-16.

7- Daniel SJ. Compliance with infection-control procedures and attitudes of oral health care providers toward patients with HIV/ AIDS: a synthesis of the literature. J Dent Hyg 1998; 72: 33-45.

8- Discacciati JA, Sander HH, De Castilho LS, Resende VL. Determination of the dispersion of microorganisms in the course of dental surgical activity. Rev Panam Salud Publica 1998; 3: 847.

9- Graham PE, Harel-Raviv M. The law and ethics in relation to dentists treating HIV-positive patients: report on a recent U. S. Supreme Court case. J Canad Dent Assoc 1999; 65: 27-30.

10- Gibson B, Freeman R. Dangerousness and dentistry: an explanation of dentists' reactions and responses to the treatment of HIV-seropositive patients. Comm Dent Oral Epidemiol 1996; 24: $341-5$.

11-Gilbert AD, Nuttall NM. Knowledge of the human immunodeficiency virus among final year dental students. J Dent 1994; 22: 228-35.

12- Kitaura H, Adachi N, Kobayashi K, Yamada T. Knowledge and attitudes of Japanese dental health care workers towards HIVrelated disease. J Dent 1997; 25: 279-283.

13- Kopacz DR, Grossman LS, Klamen DL. Medical students and AIDS: knowledge, attitudes and implications for education. Health Educ Res 1999; 14: 1-6.

14- Lopez N, Glick M, Berthold P. Providing care in an infectious disease clinic. Why students volunteer. Eur J Dent Educ 1998; 2: 138-42.

15- McCarthy GM, MacDonald JK. Nonresponse bias in a national study of dentists' infection control practices and attitudes related to HIV. Comm Dent Oral Epidemiol 1997; 4: 319-23. 
16- McCarthy GM, Koval JJ, MacDonald JK, John MA. The role of age- and population-based differences in the attitudes, knowledge and infection control practices of Canadian dentists. Comm Dent Oral Epidemiol 1999; 27: 298-304.

17- Maupome GC, Acosta-Gio E, Borges-Yanez SA, Diez-deBonilla FJ. Survey on attitudes toward HIV-infected individuals and infection control practices among dentists in Mexico City. Am J Infect Control 2000; 28: 21-4.

18-Maupomé-Carvantes G, Borges-Yáñez SA. Actitudes y costumbres para el control de infección por VIH y hepatitis B en estudiantes de odontología. Salud Publica Méx 1993: 35: 642-50.

19-Molinari JA. Dental infection control at the year 2000: accomplishment recognized. J Am Dent Assoc 1999; 130: 12918 .

20- Ogden GR, Baharami M, Sirvarajasingan V, Phillips G. Dental student's knowledge and compliance in cross infection control procedures at a UK dental hospital. Oral Dis 1997; 3: 25-30.

21-Scully C, Porter SR. Oral mucosal disease: A decade of new entities, aetiologies and associations. Int Dent J 1994; 44: 33-43.

22- Sposto MR, Gonçalves F, Ferracioli A, Porter SR, Afonso W, el-Maaytah M, Di Alberti L, Scully C. Willingness of Brazilian dentists to treat an HIV infected patient. Oral Surg Oral Med Oral Pathol 1994; 78: 175-7.

Recebido para publicação em: 02/12/2002

Aceito após reformulações em: 16/04/2003

ENDEREÇO PARA CORRESPONDÊNCIA

FACULDADE DE ODONTOLOGIA DE

ARARAQUARA - UNESP

Prof $^{\mathrm{a}}$ Dra $^{\mathrm{a}}$ Maria Regina Sposto

Departamento de Diagnóstico e Cirurgia

Rua: Humaitá, 1680 - CEP 14.801-903 - C.P. 331

tel.: (0**16) 201-6366 / 201-6359

E-mail: sposto@foar.unesp.br 